\title{
INCOMPLETE KAWASAKI DISEASE: WHAT CAN WE DO ABOUT IT?
}

\author{
LUMINIŢA DOBROTĂ ${ }^{1}$, CORINA CAZAN ${ }^{2}$, DAN-VLADIMIR BRATU ${ }^{3}$, BOGDAN NEAMŢU ${ }^{4}$ \\ 1,2,4 "Lucian Blaga" University of Sibiu, ${ }^{2}$ Pediatric Clinic Hospital, Pediatric Clinic, Sibiu, " "Carol Davila" University of Medicine and Pharmacy \\ Bucharest, Bucharest Emergency and Teaching Hospital, ${ }^{4}$ Pediatric Clinic Hospital, Pediatric Clinic-Research and Telemedicine Center in \\ Neurological Diseases in Children, Sibiu
}

\begin{abstract}
Keywords: $\quad$ Kawasaki Abstract: Kawasaki disease is a rare condition that mainly affects children younger than 6 years old. disease, incomplete form, However, it represents the most common cause of acquired heart disease and the second most differential diagnosis, frequent vasculitis in children. Its importance consists in cardiac (coronary) complications identified early diagnosis in adults younger than 40 years old. Early diagnosis is pivotal for preventing (or reducing) coronary aneurysms and avoiding, at least, later unnecessary surgical interventions. Full (classic, complete) Kawasaki disease is easily diagnosed, even if the symptoms are not always present at the same time and most of them are unspecific. Incomplete Kawasaki disease implies challenge, delay or misdiagnosis. "Picking it up early is a winner" - the specialists say, so that early treatment administered at the right moment can stop the inflammatory process leading to much better outcomes, consequently.
\end{abstract}

\section{INTRODUCTION}

Kawasaki disease is an acute febrile vasculitis, selflimited, with unknown etiology. Affected children are generally younger than 5 years old $(80 \%)$, with a peak at $9-11$ months. It involves the medium-sizem extraparenchymatous arteries, having a predilection for coronary arteries. It is more frequent in Asian and Asian-descending population, with flare-ups in winter and early spring.

The etiology remains unknown, possibly a transmissible infectious cause, with a genetic susceptibility pathogenity. A new ARN-virus (superantigen) leads to an immunologic cascade (neutrophils, IL-1, IL-6, TNF), with adaptative immune responses, both proinflammatory and regulator ones.(1)

The complete form of Kawasaki disease is defined, according to the American Heart Association guide, 2017, in the presence of unexplained fever, lasting 5 days or longer, and 4 of the following features (figure no. 1):

bilateral conjunctivitis;

lips/oral mucosa erythema;

erythemateous oedema of palms and soles;

polymorphous exanthema;

cervical lymphadenophaty.(1)

Starting from this definition, there are incomplete forms, exceptions, adaptations, in order to allow a better recognition of the disease:

- $\quad$ the Japanese Heart Society (2008) does not limit the fever duration;

- fever, 3 days or longer, and fewer than 4 features (previously stated), but supported by lack of alternative diagnosis and/or high imflammatory markers ( $\mathrm{C}$ reactive protein, ESR, neutrophilia);

- the presence of other clinical features (irritability, BCG scar inflammation, other system involvement - arthritis, pneumonitis, gastroenteritis, myocarditis, uveits, sterile pyuria);

- positive echocardiogram (coronary aneurysm), at any time, with less than 4 clinical features, already mentioned.(2)

Figure no. 1. Clinical features of Kawasaki disease (Image: Kawasaki disease Canada www.kdcanada.org)

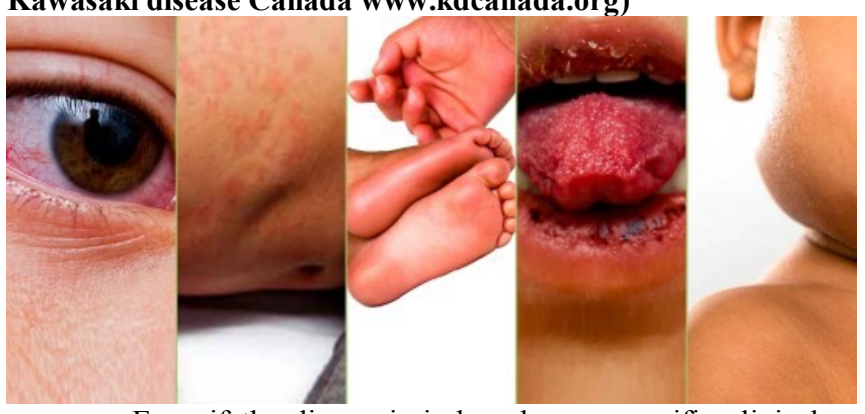

Even if the diagnosis is based on unspecific clinical signs/symptoms and some of them can spontaneously disappear, each feature has a small peculiarity. $(1,3)$

Fever, $39-40^{\circ} \mathrm{C}$, is a remittent one (morning-evening variation less than $1{ }^{\circ} \mathrm{C}$, with the lower limit never decreasing below $37^{0} \mathrm{C}$ ):

spontaneous duration 1-3 weeks;

remitted after 36 hours of treatment (but may reappear); spontaneous dissolution does not exclude the diagnosis.

Bilateral conjunctivitis ( $90 \%$ of Kawasaki forms): shortly after fever onset, painless;

complicated in the first week with uveitis (70\%), which implies photophobia, eye pain, with fast recovery.

Lips/oral mucosa erythema:

dryness, cracks, desquamation, bleeding

raspberry tongue

${ }^{1}$ Corresponding author: Luminița Dobrotă, Str. Lucian Blaga, Nr. 2A, Sibiu, România, E-mail: luminitadobrota@yahoo.com, Phone: +40722501145 Article received on 20.08.2020 and accepted for publication on 15.12.2020 


\section{CLINICAL ASPECTS}

- diffuse erythema of oro-pharingeal mucosa; but never, pharingeal ulcers or exudate.

Erythemateous oedema of palms and soles: the last feature of acute stage;

- $\quad$ peeling, in subacute stage (nail, than palms and soles), very specific $(100 \%)$;

- $\quad$ Beau lines, after 1-2 months (figure no. 2).

Figure no. 2. Beau lines (Leukonychia striata in Kawasaki disease; J Pediatr 2008,152:889)

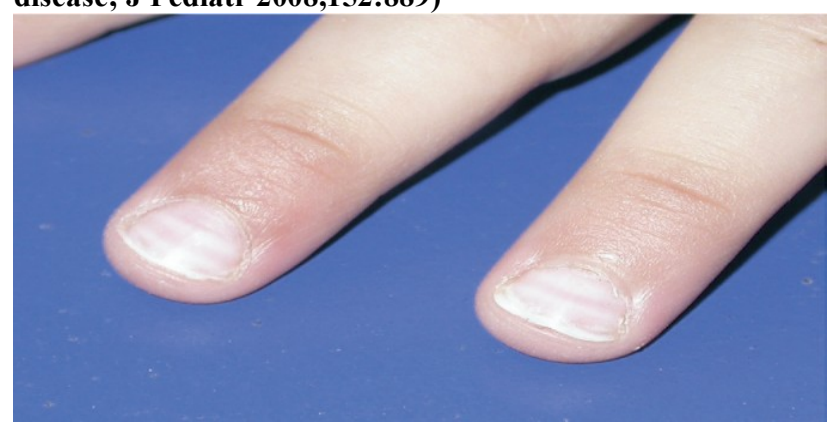

Polymorphous exanthema:

in the first 5 days after fever onset;

- typical onset in the perineal region (with early desquamation), extending to the trunk and limbs;

types:

diffuse maculopapular;

scarlet fever-like;

multiform erythema;

atopic dermatitis (in subacute stage);

micropustules (rare);

psoriasis (rarely);

never bubbles, vesicles, petechiae.

unilateral.

Cervical lymphadenopathy

- painless.

The laboratory findings are unspecific too:

- neutrophilia.

- $\quad$ high platelet count (characteristic!), 2 weeks after disease onset (with a peak -700.000 up to $1.000 .000,3$ weeks after onset).

- $\quad$ elevated inflammatory markers (C reactive protein, ESR, ferritin).

- $\quad$ low albumin (produced by capillary loss, not by hepatic cytolysis).

dyslipidemia.

low haemoglobin.

moderate hepatic cytolysis;

high conjugated bilirubin;

low sodium.(4)

"Once seen, never forgotten", but Kawasaki disease is still a diagnosis that can be easily missed.(4) Because of unspecific clinic and laboratory findings, the list of differentials can be exhaustive (table no. 1)

Table no. 1. Differential diagnosis in Kawasaki disease

\begin{tabular}{|l|l|}
\hline Retropharingeal abscess & Scarlet Fever \\
\hline Peritonsillar abscess & Rheumatic fever \\
\hline Cervical lymphadenitis & Toxic Shock Syndrome \\
\hline Group A streptococcal infection & $\begin{array}{l}\text { Staphylococcal scalded skin } \\
\text { syndrome }\end{array}$ \\
\hline $\begin{array}{l}\text { Adenovirus, Enterovirus, } \\
\text { Parvovirus B19 }\end{array}$ & Toxic epidermal necrolysis \\
\hline Measles & Lyme disease \\
\hline Mononucleosis & Leptospirosis \\
\hline Drug reaction & Etc. \\
\hline
\end{tabular}

Since Kawasaki disease requires treatment no later than 10 days from onset (fever onset), the exclusion of the differentials involve an even longer period of time.

Kawasaki disease must be considered in case of:

an infant younger than 6 months old with prolonged fever and irritability;

an infant with prolonged fever and unexplained aseptic meningitis (cerebrospinal fluid with pleocytosis and negative culture, changes often associated with "beheaded" meningitis);

- $\quad$ an infant with prolonged fever and unexplained or culturenegative shock;

- an infant or child with prolonged fever and cervical lymphadenitis unresponsive to antibiotic therapy;

an infant or child with prolonged fever and retropharyngeal phlegmon unresponsive to antibiotic therapy

an infant with prolonged fever and pyuria followed by rash (interpreted as antibiotic reaction). $(4,5)$

The unspecific laboratory findings can create confusions and delay the appropriate start time of treatment: $(1,2)$

- Kawasaki disease is unlikely if C reactive protein, ESR and platelet remain normal after 7 days of disease; but this is how most viral infections behave, at least at the onset or in the absence of complications;

- low neutrophil count and lymphocyte predominance suggest an alternative diagnosis;

- leukocytosis, with granulocyte predominance, is typical in the acute stage; but this is how most bacterial infections present;

- normocytic, normochromic anemia is common during (unspecific) inflammation (see, figure no. 3); no doubt, but for infants, at the least, the most frequent anemia which pediatricians find in usual practice is microcytic, hypochromic;(6)

Figure no. 3. The mechanism of anemia and its role in coronary aneurysm formation: the toll-like receptors upregulate the hepcidin expression; after hepcidin interacts with ferroportin, the last one leads to iron sequestration and iron inhibition of duodenum absorbtion; hepcidin also inhibits erythropoiesis, which leads to transient hyposodemia and anemia; IvIg significantly decrease the hepcidin level.(6)

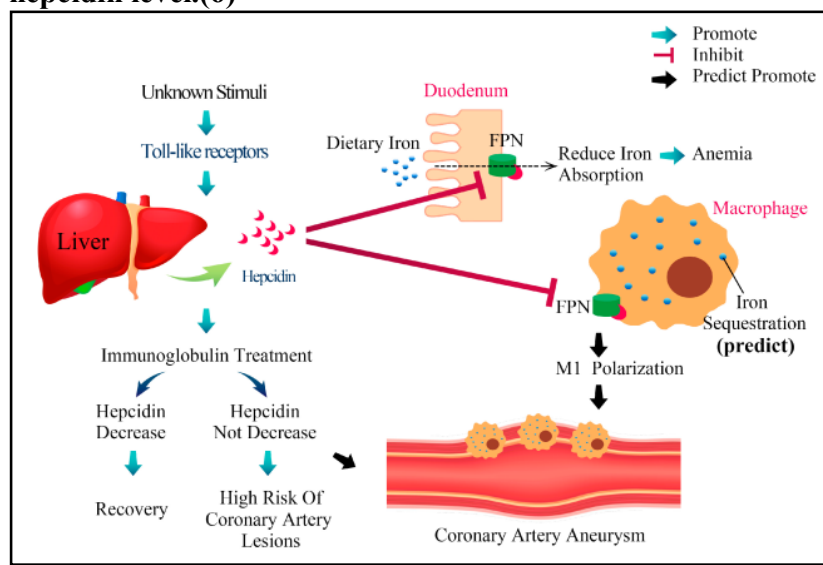

$\mathrm{C}$ reactive protein normalized more quickly with inflammation resolution; note that $\mathrm{C}$ reactive protein normalizes quicker by IvIg therapy, opposite with ESR which is elevated by IvIg therapy;

minimally elevated ESR in the setting of severe clinical disease should prompt investigation for disseminated intravascular coagulation; most of severe disease behaves the same; 


\section{CLINICAL ASPECTS}

- thrombocytosis, the characteristic feature, generally doesn `t occur until the second week, peaking in the third week and normalizing by 4 to 6 weeks; limited utility for early diagnosis;

- thrombocytopenia can be a sign of disseminated intravascular coagulation (universal sign) and is a risk factor for the development of coronary artery aneurysms; mild to moderate elevations in serum transaminases or gammaglutamyl transpeptidase occur in $40-60 \%$ of patients, and mild hyperbilirubinemia occurs in almost $10 \%$; being moderate forms can be interpreted in the context of many kind of infections or as an effect of therapy (antibiotics, etc.)

- hypoalbuminemia is common and associated with more severe and more prolonged acute disease; true, but albuminemia is not mandatory in usual practice; is utilized in particular cases;

- $\quad$ urinalysis may show pyuria in up to $80 \%$ of cases, but is non-specific for Kawasaki disease;

- $\quad$ not in the least, the presence of streptococcus in pharingeal swabs or an elevated $\mathrm{O}$ antistreptolysine can create confusion between differentials (scarlet fever or not, even more so because the age of scarlet fever onset decreased in recent years); if in doubt, it is better to treat both.

Once the differential diagnosis has been made, and all the mandatory laboratory tests done, great importance has to be given to the echocardiogram. This type of investigation needs to be carried out, but without delaying treatment and can be done even after treatment has been started. On the one hand, the echocardiogram gives meaning to Kawasaki disease diagnosis (for incomplete form) and makes it easier to start the treatment, but on the other hand the role of treatment (IvIg, mainly) is to "switch off" the inflammatory process, minimising damage to the vessels and, hopefully, preventing coronary aneurysms from developing. Finally, it reduces the risk of coronary artery aneurysms from 25 to $5 \%$. Coronary aneurysms leads to an increased risk of thrombosis and myocardal ischemia/infarction. It is also possible for aneurysms to rupture, causing cardiac tamponade.(7)

Children who have myocardial ischaemia can present with typical adult type symptoms: chest pain, shortness of breath or pallor/looking unwell; however they can also present with other features such as unexplained crying, tachycardia, abdominal pain or tachypnoea.

The natural course of Kawasaki disease in the subacute stage consists of a peak in mortality when the presence of coronary aneurysms is combined with thrombocytosis and hypercoagulability state. Death by myocardial ischaemia/infarction occurs much later (adults less than 40 years old).(1,2)

(Coronary) aneurysms are defined as vessels with a diameter of more than $3 \mathrm{~mm}$ in patients younger than 5 years old and more than $4 \mathrm{~mm}$ in patients older than 5 years. Later in adulthood cardiac complications are more common in case of aneurysms greater than $6 \mathrm{~mm}$.(7)

The echocardiogram must be performed at the time of diagnosis, 2 weeks after symptom (fever, especially) onset, and at 8 weeks after. American Heart Academy recommends repeat echocardiogram 10-14 days after the first one and 4-6 weeks after all the laboratory analysis have normalized.(8)

The risk of coronary aneurysm is higher in the following situations:

- $\quad$ fever lasting more than 8 days (the most important one);

- $\quad$ fever recurrence after a 48 hours afebrile period;

male gender (3 times more affected for giant aneurysms);

cardiomegaly;

less than 1 year old of age;
Asian and Pacific islander descent;

hispanic ethnicity;

lower level of IgG;

higher level of TNF-alpha;

thrombocytopenia, at the onset of disease;

incomplete Kawasaki disease. $(4,5)$

Even more, in this kind of situations, the management of diagnosis and treatment should be started as soon as possible (the earlier management, the better the outcome).

\section{CONCLUSIONS}

Kawasaki disease is a rare condition, with difficult diagnosis, even more so for its incomplete form. The incomplete form represents by itself a risk factor for coronary aneurysm. Thus, all clinical (not only those from classic form) and biological criteria (of diagnosis) must be well-judged, and the treatment quickly established, for a good outcome, finally.

\section{Acknowledgement.}

Part of the analysis has been conducted in the Pediatric Clinic Hospital Sibiu, within Research and Telemedicine Center in Neurological Diseases in Children CEFORATEN project (ID928 SMIS-CSNR 13605) financed by ANCSI with the grant number 432 / 21. 12. 2012 thru the Sectoral Operational Programme ,Increase of Economic Competitiveness".

\section{REFERENCES}

1. Liu YC, Lin MT, Wang JK, Wu MH. State of the art acute phase management of Kawasaki disease after 2017 scientific statement from American Heart Association. Pediatrics and Neonatology. 2018;59;543-552.

2. Diagnosis, treatment, and long-term management of Kawasaki disease. Circulation. 2017;135:e927-999.

3. Iordachescu F. Tratat de Pediatrie. Editura ALL; 2019.

4. Saguil A, Fargo M, Grogan S. Diagnosis and management of Kawasaki disease. American family physician. 2015 Mar 15 [PubMed PMID: 25822554].

5. Lang B, Recognizing Kawasaki disease. Paediatrics [PubMed PMID: 20084135].

6. Huang YH, Kuo HC. Anemia in Kawasaki Disease: Hepcidin as a Potential Biomarker. Int J Mol Sci. 2017;18(4):820;https://doi.org/10.3390/ijms18040820.

7. Denby KJ, Clark DE, Markham LW. Management of Kawasaki disease in adults. Heart (British Cardiac Society). 2017 Nov [PubMed PMID: 28751537].

8. Tsuda E, Singhal M, Role of imaging studies in Kawasaki disease. International journal of rheumatic diseases. 2018 Jan [PubMed PMID: 29115035]. 